\title{
The Use of Hypermedia in Group Problem Solving: An Evaluation of the DOLPHIN Electronic Meeting Room Environment
}

\author{
Gloria Mark, Jörg M. Haake, Norbert A. Streitz \\ IPSI - Integrated Publication and Information Systems Institute \\ GMD - German National Research Center for Computer Science \\ Dolivostr. 15, D - 64293 Darmstadt, Germany \\ \{mark, haake, streitz\}@darmstadt.gmd.de
}

Abstract

In this paper, we report on an empirical evaluation of selected aspects of DOLPHIN, a meeting room environment of computers networked with an electronic whiteboard. Our results show that in a face-to-face meeting, the use of DOLPHIN's hypermedia functionality changed the nature of the product and the way groups worked, compared to using only electronic whiteboard functionality. Groups organized their ideas into network, rather than pure hierarchical, structures. These were more deeply elaborated, contained more ideas, and had more relationships between the ideas. The problem solutions were also judged to be more original. Groups were more likely to use a top-down planning strategy, and to exhibit a different temporal work pattern. The results suggest that work groups can benefit from using hypermedia in problem solving.

\section{Introduction}

The design of DOLPHIN is grounded in a conceptual framework which tries to bring together the research areas of CSCW and hypermedia. While a wide range of collaborative work can benefit from the approach taken, one can characterize our starting point as support for document-based activities, i.e., work where documents are either produced or are used as means during group work. 
In our approach, we use the notion of "documents" in a very general and comprehensive way. Documents are not only traditional memos, letters, articles, or books, but they also include sets of scribbles and drawings on a whiteboard or an overhead transparency, and collections of information items which can contain multimedia elements (pictures, audio, video) which are usually available in electronic formats. In general, we consider documents to be hypermedia documents. This implies the possibility of employing nonlinear network structures of complex relationships (links) of information components (nodes) as it is defined by the concept of hypertext (Nielsen, 1995). We consider hypermedia as the subject matter to be created and used as well as a medium facilitating computer-supported cooperative work (Streitz, 1994). Defining relationships between different tasks, coordinating and assigning tasks to different group members, commenting and communicating on the progress of parts of the overall work activities, and much more, can be represented as hypermedia structures. At the same time, this information can be linked to the (hyper)documents which are created and used in the course of group activities.

From a CSCW perspective, we currently focus on the support for face-to-face meetings and their equivalents facilitated by telecooperation techniques resulting in distributed "virtual meetings". Observing the central role of public displays as they are provided by overhead projectors and whiteboards in face-to-face meetings, we investigate what kind of added value could be provided by using an electronic, i.e. a computer controlled, whiteboard. Another line of research for computer support in face-to-face meetings focuses on providing a computer to every participant in the room. Using common application software, each participant can actively enter, edit, and use information while sitting in the meeting room. Our approach is based on recognizing that a combination of these two scenarios is needed, i.e., an interactive electronic whiteboard and computers for each participant. As group activities are dynamic, rather than static, their support requires a high degree of flexibility. Flexibility can be provided in many ways. It is our view that hypermedia systems are able to provide this flexibility and serve as an ideal basis for building the next generation of cooperative information systems.

From a hypermedia perspective, flexibility translates into investigating which class of hypermedia structures should be provided for supporting group work. A prominent aspect is the modularity and inherent annotation capability of hypermedia structures. Hypermedia allows for flexible decomposition, restructuring, and reuse of components in a dynamic fashion. Decomposition can be used for domain structuring as well as for structuring the division of labour in the group. Another aspect of flexibility refers to the degree to which a system is able to provide a wide range of structures in accordance with the requirements of the tasks, their coexistence and means for transformation (Haake et al., 1994). Combining these requirements, we propose to provide a basic node-link hypermedia model for structure representation. In addition to and coexistent with, we support more informal ways of communication by "free form" scribbling, drawing, gesturing, etc. as is the case on (electronic) whiteboards. Since DOLPHIN is a cooperative hypermedia system, this range of flexible structures 
can be used by each group member simultaneously at the workstation and/or at the public electronic whiteboard. DOLPHIN's collaboration functionality provides shared workspaces between all group members with an additional distinction between private and public workspaces (Streitz et al., 1994).

The evaluation of DOLPHIN is concerned with a unique combination (to our knowledge) of physical setting and cooperative hypermedia functionality. Nevertheless, we can relate our approach to forerunners in the development of relevant technologies. Due to space limitations, we cannot discuss these systems in detail. These include CSCW environments such as CoLab (Stefik et al., 1987), the Capture Lab (Mantei, 1988) and the NICK project (Rein \& Ellis, 1989). A recent example meeting our criteria of an electronic whiteboard is the LiveBoard (Elrod et al., 1992), in combination with appropriate software, e.g., Tivoli (Pedersen et al., 1993). Software supporting cooperative activities include GroupSystems (Nunamaker et al., 1991) and ShrEdit (McGuffin \& Olson, 1992). We can also include systems primarily used in telecooperation situations, e.g., ClearBoard (Ishii et al., 1993), cooperative hypermedia systems, e.g., DHM (Grønbaek et al., 1994), SEPIA (Streitz et al., 1992), or collaboration in distributed design (Marmolin et al., 1991).

\section{Motivation and goals for the evaluation experiment}

In order to obtain relevant feedback for the revision of the current design of DOLPHIN, we planned an evaluation experiment addressing basic design decisions, especially related to the support of group problem solving. Since we consider the role of hypermedia structures to be highly relevant for the design of CSCW systems, we focused the experiment on the effects of adding hypermedia functionality to what can be considered to be standard on an electronic whiteboard. We chose to study the effect of adding a 'simple' node-link model (see section 3). This is motivated by our focus on supporting processes especially in the early phases of group problem solving such as problem exploration, idea generation, information structuring, and adding/reducing information elements.

We propose that the use of hypermedia structures is beneficial because it provides multiple ways and parallel views of a problem representation. Psychological evidence suggests that the quality or accuracy of the problem solution is dependent on the appropriateness of the external problem representation (Newell, 1980, Schwartz, 1971, Streitz, 1985) and that the problem solving process is facilitated by opportunities for multiple views and representations of the problem structure (Gick \& Holyoak, 1983, Mayer \& Greeno, 1975). We investigated whether DOLPHIN's hypermedia functionality compared to DOLPHIN without hypermedia functionality could yield a different problem representation in terms of how information is structured. This resulted in two conditions of the experiment: our implementation of more or less standard electronic whiteboard functionality vs. this functionality in combination with additional hypermedia structure functionality. We concentrated on the early phases of group problem solving. 
In the context of this experiment, this led us to address the following questions which again determined our choice of dependent variables and measures.

1. Hypermedia document creation: for groups as well as for individuals?

While there is evidence that users with much experience can create very extensive hypermedia documents over time (Landow, 1989, Schuler et al., 1995), it is not yet clear whether users without much experience and situated in a face-to-face meeting can also portray their ideas in a hypermedia format within a reasonable training time. This might become even more critical if the composition of the group is more or less ad hoc as it might happen, e.g., in a business environment.

2. Does hypermedia facilitate the formation and elaboration of relationships?

The hypermedia functionality of creating nodes and links enables users to form multiple connections between concepts, i.e., many-to-many mappings. We would expect that this functionality would facilitate the creation of a network structure which we define as a structure containing at least one concept with multiple connections to other concepts. We would expect this as opposed to a pure hierarchical structure with superordinates at the highest level and subordinate concepts at lower levels (no cross-hierarchical connections). On the other hand, we expect that using a standard electronic whiteboard where links are not provided as a system feature (although subjects can handdraw arrows to express relationships) would not necessarily lead one to create a network structure.

Based on the property that hypermedia node structures can be used to elaborate ideas by using nested nodes (each containing content), we expect that this functionality would lead groups using hypermedia structures to elaborate their ideas more, creating subsequently deeper levels containing new information compared to groups using only a flat electronic drawing surface. There might also be a tradeoff between depth and breadth. The nested nodes, along with the link functionality should result in more ideas associated with a superordinate concept compared to a nonhypermedia information structure.

3. How does hypermedia influence strategy and memory?

We expect that the role of hypermedia in group problem solving will also be reflected in the strategies used and in the retention of information. Working with hypermedia may lead groups to fundamentally change their planning strategy when organizing ideas.

According to the depth of processing view of memory (Craik \& Lockhart, 1972), information that is more deeply processed should be reflected in a better memory of the information. Therefore, we expect that the decision-making process of turning information into nodes should result in deeper processing of the information, and would be reflected in a better memory of the ideas compared to groups not using hypermedia structures. In addition, we expect that the higher the level of the hyperdocument structure, the better would be the memory of the information at that level due to repeated exposure, importance of information, and strength of association (Wingfield \& Byrnes, 1981).

4. How does hypermedia structuring influence group participation?

The possibilities of decomposition by using hypermedia structures can be used for the distribution of tasks among group members and for parallel work and may 
affect group participation. We expect that different decision making occurs when organizing information into a hypermedia structure and it may be reflected in differences in group participation, such as speaking or system use.

\section{The DOLPHIN System}

\subsection{Hypermedia functionality}

DOLPHIN provides scribbles, text, nodes and links. A scribble is freehand writing or drawing, such as exclamation marks, Figures like boxes, circles, arrows, tables, etc. A text is a string of ASCII characters.

DOLPHIN documents begin with a top node corresponding to the highest level of the document. Nodes consist of a title and a content. The content of a node can contain scribbles, text, and links to other nodes. A content of a node is displayed in a DOLPHIN window. DOLPHIN provides operations for creating, editing, selecting, moving, copying, pasting and deleting scribbles, text, nodes and links.

Links connect different nodes. DOLPHIN supports two kinds of links: internode links for navigational purposes and intra-node links as a kind of graphical representation of relationships between nodes. Inter-node links start in the content of a node and end in the content of another node. They are displayed in the source content by a node representative for the destination node (thus, functioning as anchors). Following the inter-node link leads to the content of the destination node and displays it in a new DOLPHIN window. Users can create such a link either by creating a new node and including its representative in the currently displayed content, or, they can copy an already existing node representative and paste it into a different content. In hypermedia systems, these links are usually called embedded links. With inter-node links users can create hierarchical as well as non-hierarchical structures between nodes. Intra-node links connect two nodes contained in the same content. Users can create these links between any two node representatives in the same content. These links present themselves as directed arrows. In hypermedia systems, they are usually called node-to-node links.

Using the above types of objects and operations, users may create different structures, ranging from hierarchically nested structures, i.e. each node at a higher level of the hierarchy contains the nodes of the next lower level (thus forming tree-like structures) to nonlinear structures where nodes are included in the content of several other nodes (thus constituting nonlinear graph structures). Users can also create graphical arrangements of objects in each node's content (see Figure 1).

The pen-based user interface of DOLPHIN provides gestures for creating, deleting, moving, and selecting objects and for opening a node's content. An always visible menu with buttons for cut-copy-paste operations, closing DOLPHIN windows, and erasing scribbles is provided by DOLPHIN windows. 
These objects are mapped onto a general hypermedia data model provided by an underlying cooperative hypermedia server. Each object includes their content and additional presentation attributes (e.g., size, color, position). Further technical information can be found in Streitz et al. (1994) and Haake et al. (1994).

\subsection{Cooperative editing and viewing functionality}

When working with DOLPHIN, users can share the content of a node, thus using it as a public workspace which displays all nodes, links, text and scribbles at that level. They can also decide to work in different nodes, thus each using a private workspace. Within a shared workspace, DOLPHIN supports concurrent operations performed by different users. Shared access and active update/synchronization of concurrent DOLPHIN windows displaying the same node's content are provided by DOLPHIN's cooperative hypermedia server. All changes to the DOLPHIN hypermedia document are reflected in the hypermedia server and made persistent.

\section{Method of the Experiment}

\subsection{Setting}

In our electronic meeting room (the OCEAN Lab), groups are seated around a rectangular table with a large interactive, electronic whiteboard facing them on one end of the table. While other scenarios are possible (cf. Streitz et al., 1994), in this experiment, DOLPHIN was used only to support two usages: as a pen-based single user system on an electronic whiteboard and as a multi-user application shared between an electronic whiteboard and networked computers.

In this particular experiment, we used a SUN-based Xerox Liveboard (Elrod et al., 1992) as the hardware basis for the electronic whiteboard and two SUN Sparc2 workstations with 17 " color monitors mounted into the meeting table. One subject worked on the Liveboard, visible to everybody. Two subjects used the workstations while still being able to see and talk to the others. The public workspace on the Liveboard could also be presented on the workstations. For the public displays, we provide a relaxed WYSIWIS functionality (What You See Is What I See; Stefik et al., 1987), which means that users could scroll and resize their windows independently. The provision of workstations to two subjects allowed them to work in parallel in a "private workspace" (similar to taking individual notes on paper or looking at separate documents, etc.).

\subsection{The evaluation infrastructure}

During the experiment, various data have been recorded. Screendumps were taken from every workstation and the Liveboard every 15 seconds by a snapshot program. The total view of the meeting room was videotaped. The final state of the meeting document was automatically captured in DOLPHIN's cooperative 
hypermedia server. After the meeting, the document structure was reconstructed on a piece of paper and coded. An observer took notes during the meeting.

To be able to evaluate concurrent activities of several users using the computers provided in the meeting room, a specialized evaluation tool has been developed. The screenshots from the workstations and the Liveboard plus the videotape were digitized into Quicktime movies on a Macintosh computer. The evaluation tool can present all four digital video streams concurrently on the Macintosh. One may choose to play one or all of the four streams available, resize the active video window, hear the sound in parallel to the video, and synchronize the screen content of all participants. By playing the video streams backwards or forwards, one can observe how the meetings developed. This tool was especially valuable for analyzing the groups' strategies during the meetings.

\subsection{Design}

In order to isolate the effects of using hypermedia structures, a between-subjects design was used. Subjects worked in groups of three, with eight groups per condition. Groups were assigned to two conditions which correspond to two different functionalities of the DOLPHIN system:

Nonhypermedia structures (N-Condition). Subjects were trained only in the nonhypermedia structures which employs the standard electronic whiteboard functionality. Here, DOLPHIN offers scribbles with the pen (and mouse) and typed text using a keyboard. These are objects which can be created, selected, moved, and deleted. Subjects could work only in one window, but could scroll to any area of the window and could scroll to different views independently from each other if they preferred.

Hypermedia structures (H-Condition). Subjects were trained both in the nonhypermedia structures of DOLPHIN (see above) as well as in the hypermedia structures of the system: for nodes and links the operations included creation, selection, opening (of nodes), deletion, and movement. Subjects were also shown how to create nonlinear structures by copying and pasting nodes and links.

\subsection{Subjects}

The experiment took place during an eight-week period in Fall of 1994. A total of 48 subjects were mainly recruited from students of the Technical University in Darmstadt, while eight of them were recruited from the staff of GMD-IPSI, most of whom had received their university degree within three years. Subjects were assigned to conditions so that students and staff were randomly distributed among the groups. Subjects were not paid. No significant differences were found in subjects' computer experience, or age, between the conditions.

\subsection{Procedure}

Subjects first received a 40-minute training session on the system functionality. In both conditions, subjects were shown an example of a network structure on the Liveboard as a possible information structure that one can create with the 
DOLPHIN system. In the N-Condition, the structure was created with handwritten words and arrows; in the H-Condition, the same structure was created with nodes and links. Afterwards, subjects worked on a 20-minute practice exercise.

After training, groups were instructed to spend the first 20 minutes in brainstorming and were read standard instructions for this task (Osborne, 1957). Subjects then were told to spend 40 minutes structuring and developing their brainstormed ideas. In both conditions, subjects were told that they could order their ideas into categories or graphical arrangements. In addition, subjects in the $\mathrm{H}$-Condition were told that they could use nodes and links to structure their ideas. It was emphasized that subjects in both conditions could structure their ideas any way they preferred, using their own strategy, and were not bound to any particular format. Subjects could choose to work in any style they wanted: together, separately, or varying their styles. Directly after the experiment, subjects were given a memory test and then filled out a questionnaire in order for us to survey users' work group experience, opinions of the system, and to receive suggestions.

\subsection{Task}

It was decided that the task should meet the following requirements: 1) it should have the potential to allow subjects to generate a wide range of ideas, 2) its solution should be able to comfortably assume both the form of a nonhypermedia and hypermedia structure, 3) it should have a realistic purpose to increase motivation, and 4) it should be complex enough that its solution could take advantage of DOLPHIN's electronic capabilities in both experimental conditions.

Subjects were told to design a "library of the future" for the city of Darmstadt, Germany. They were to prepare a proposal for the city, which did not have to be in the form of a finished text document. The instructions stated that the citizens of Darmstadt would be the main users, cost was not to be a factor, financing should be left out, and ideas were to be generated without consideration of their feasibility, i.e. that current technology did not have to exist to realize their ideas.

\subsection{Measures}

The choice of our measures was guided by the hypotheses that we earlier developed addressing hypermedia use:

Relationship between ideas: The information structures produced by both conditions were categorized into network, pure hierarchical, and other.

Elaboration of superordinate concepts: We counted the number of levels (depth) in the documents in both conditions. Depth of the document is defined as the deepest pathway that one can reach beginning from the highest level of the document. In the H-condition it was the top node, and in the $\mathrm{N}$-condition it was the highest level which in all cases was identifiable by some graphical marker such as an underline or enumeration. We also measured breadth, defined as the number of superordinate concepts at the highest level of the document.

Ideas connected to superordinate pathway: We counted the average number of ideas following intra- and inter-node links along each superordinate pathway. 
Changes of ideas from brainstorming to information structuring. We counted how many ideas were generated during brainstorming and the net number of ideas the group ended up with after structuring the ideas, after additions and deletions. Each node was counted as one idea, and details in parentheses were included as part of the same idea.

Quality of documents. We asked two experts, a linguist, and a sociologist specializing in conversation patterns, to judge the documents along four dimensions. Logic of local structure was a measure of the logical relationship of elements within separate parts of the document (nodes in the hypermedia document, and clusters in the nonhypermedia document); logic of global structure was a measure of the logical relationship between separate parts of the document; originality of solution was a measure of how inventive/unusual were the ideas, the general approach to the solution, and the framework that the ideas were presented in; and comprehensiveness was a measure of how multifaceted the document was.

Group Strategy. Using the evaluation tool, groups' strategies were categorized as: primarily top-down (all/most of the superordinate concepts are set up initially; subordinate concepts are then categorized under them), depth-first (one superordinate concept was set up, developed with subordinate concepts, and then the process repeated for each superordinate), primarily bottom-up (subordinate concepts are first grouped, and then superordinate concepts assigned), and mixed (a mixture of the preceding strategies).

Memory of information. Directly after the experiment, subjects were given 15 minutes to try to reconstruct with paper and pencil the ideas and structure which they had just created.

The effect of hypermedia structuring on group processes was measured by:

Group participation. The length of time in seconds of speaking and of system use by each group member was coded from the videotapes.

Group satisfaction. Satisfaction was measured in the questionnaires.

\subsection{Coding}

For all dependent measures that we report, with the exception of group participation, coding was done by two separate coders, with percentage agreement exceeding $89 \%$ (this value occurred with quality of documents). For group participation, we used one coder, since the coder had to simply observe start and end times of talking and typing, and we felt that this was an objective measure that could be done competently by one coder. Where there was any question about the data, the data was checked by a second coder.

\section{Results}

Relationship between ideas. It was expected that groups in the H-Condition would be more likely to create a network structure than a pure hierarchical structure. This was indeed the case: 6 out of 8 groups in the H-Condition created a network 
structure using intra-node links whereas 7 out of 8 groups in the $\mathrm{N}$-Condition created a pure hierarchical structure $\left(\right.$ chi-square $\left._{(1)}=6.36, \mathrm{p}<.025\right)$.

Figure 1 shows a network structure created by an N-group. When H-groups did create a pure hierarchical structure, in one case they created a two-level (superordinate and subordinate) hierarchy, and in one case, they created multiple levels (four). Figure 2 shows an example of a network structure created by an $\mathrm{H}$ group. When $\mathrm{N}$-groups created a pure hierarchical structure, in six cases they created a two-level hierarchy, and in only one case did they employ three levels .

Elaboration of ideas. It was expected that subjects in the H-Condition would be more likely to create structures having deeper levels. H-groups did create structures having deeper levels and the result was highly significant $\left(\operatorname{mean}_{\mathrm{H}}=3.5\right.$ levels, s.d. $=.76$, mean $_{\mathrm{N}}=2.25$ levels, s.d. $\left.=.46 \mathrm{t}(14)=3.99, \mathrm{p}<.001\right)$. However, no significant difference was found in the breadth of the structures: $\left(\operatorname{mean}_{\mathrm{H}}=4.5\right.$ concepts, s.d.=1.85, mean $_{\mathrm{N}}=5.4$ concepts, s.d.=.74).

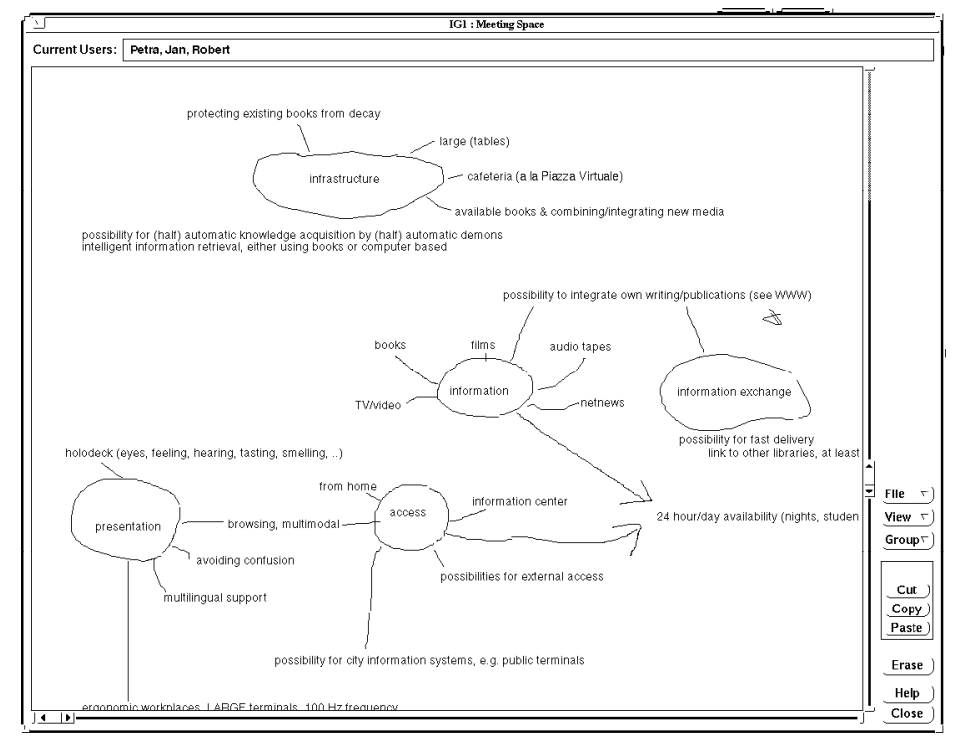

Figure 1: Example of a network structure created by an N-Group (translated from German).

Ideas connected to superordinate pathway. It was also expected that more ideas would be associated with each superordinate concept in the H-Condition. Compared to the $\mathrm{N}$-Condition, a strong trend showed that the information structures of groups in the H-Condition had, on the average, double the number of subordinate ideas connected to each superordinate concept, $\left(\operatorname{mean}_{\mathrm{H}}=13.26\right.$ ideas, s.d. $=10.08$, mean $_{\mathrm{N}}=6.60$ ideas, s.d. $\left.=2.70, \mathrm{t}(14)=1.81, \mathrm{p}<.09\right)$.

Ideas connected to superordinate pathway. It was also expected that more ideas would be associated with each superordinate concept in the H-Condition. Compared to the $\mathrm{N}$-Condition, a strong trend showed that the information structures of groups in the H-Condition had, on the average, double the number of subordinate ideas connected to each superordinate concept, $\left(\operatorname{mean}_{\mathrm{H}}=13.26\right.$ ideas, s.d. $=10.08$, mean $_{\mathrm{N}}=6.60$ ideas, s.d. $\left.=2.70, \mathrm{t}(14)=1.81, \mathrm{p}<.09\right)$.

Changes of ideas from brainstorming to information structuring. More ideas were generated by the $\mathrm{H}$-Condition than the N-Condition during brainstorming, 
but the difference did not reach significance. However, the difference increased in value over the duration of the experiment as a result of adding and deleting ideas leading to a difference which now approached significance $\left(\right.$ mean $_{\mathrm{H}}=48$ ideas, s.d. $=9.35$, mean $_{\mathrm{N}}=38.25$ ideas, s.d. $\left.=10.90, \mathrm{t}(14)=1.92, \mathrm{p}<.08\right)$.

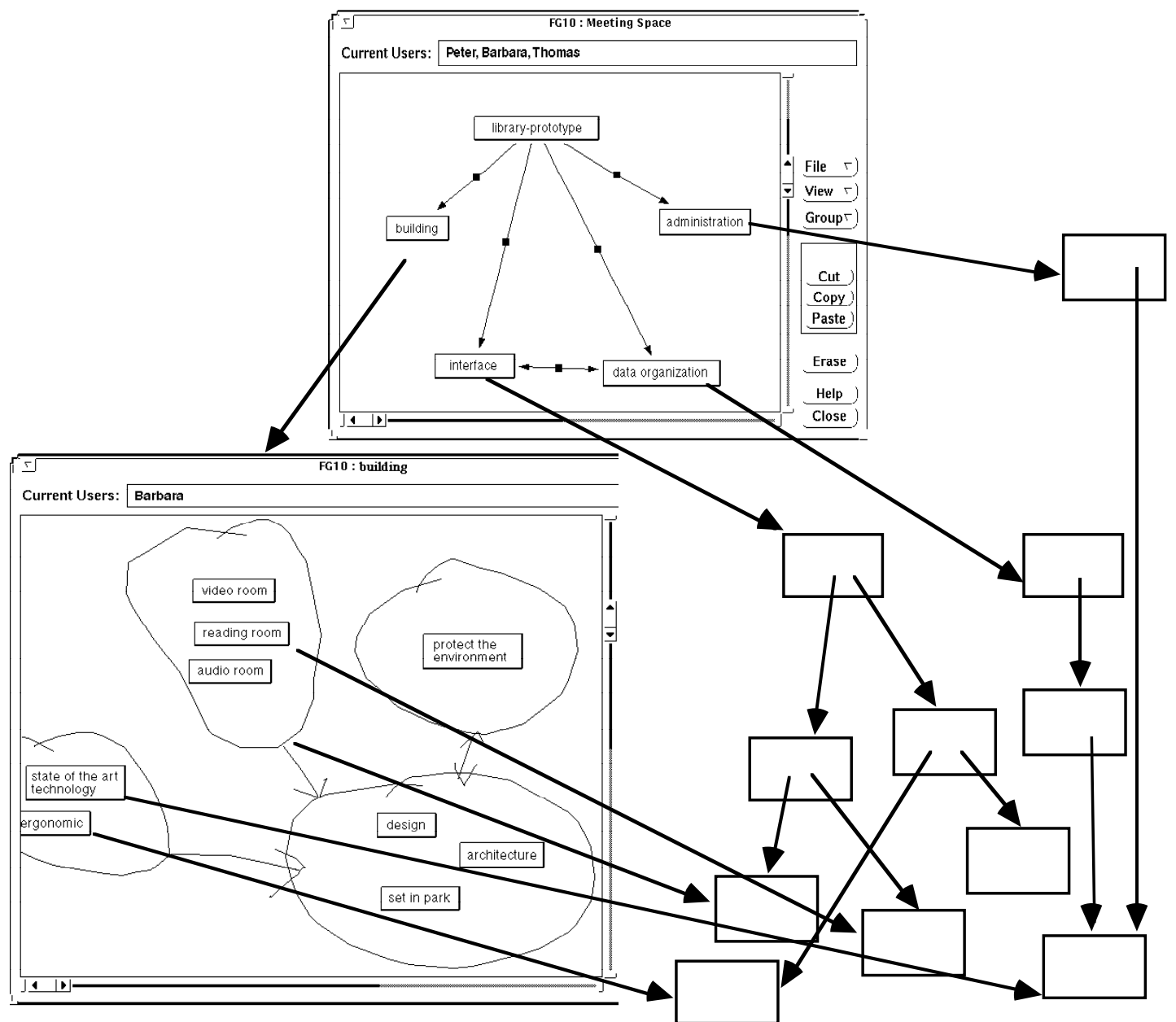

Figure 2: Results of a H-Group (translated from German). This Figure shows the content of the top node and one of the subordinate nodes. Due to lack of space we used a graphical representation of the rest of the document's structure. Here, empty rectangles denote nodes and fat arrows denote inter-node links.

Quality of documents. No difference was found by the expert coders in their judgment of the logic of local structures, logic of global structure, or comprehensiveness. However, documents from the $\mathrm{H}$-Condition were judged as being significantly more original in their solution $(\mathrm{t}(14)=2.16, \mathrm{p}<.05)$.

Quality of documents. No difference was found by the expert coders in their judgment of the logic of local structures, logic of global structure, or comprehensiveness. However, documents from the H-Condition were judged as being significantly more original in their solution $(\mathrm{t}(14)=2.16, \mathrm{p}<.05)$.

Group strategy. The type of strategy used in structuring the information was compared between conditions. Six out of eight groups in the H-Condition used a primarily top-down strategy. In contrast, groups in the N-Condition were found to 
be more likely to use a depth-first strategy ( 5 out of 8 groups). For the statistical test, a top-down vs. non top-down strategy was compared and the results show a significant difference in top-down strategy use (chi-square $(1)=4, \mathrm{p}<.05)$.

Memory of information. We expected that a higher proportion of ideas in the $\mathrm{H}$-Condition would be remembered than in the N-Condition. Surprisingly, the contrary turned out to be the case: subjects in the $\mathrm{N}$-Condition actually remembered significantly more ideas $\left(\operatorname{mean}_{\mathrm{H}}=49 \%\right.$, s.d. $=7 \%$, mean $\mathrm{N}_{\mathrm{N}}=64 \%$, s.d. $=7 \%, \mathrm{t}(13)=4.11, \mathrm{p}<.001)$. One outlying value from the $\mathrm{H}$-Condition had to be eliminated. However, subjects in both conditions remembered the same proportion of superordinate concepts $\left(\operatorname{mean}_{\mathrm{H}}=86 \%\right.$, s.d. $=19 \%$, mean $_{\mathrm{N}}=86 \%$, s.d. $\left.=16 \%\right)$. We also expected that for the H-Condition, the memory of information would increase with the document level. The results confirmed this: subjects remembered $79 \%$ of the information on the first level of the document, $42 \%$ on all intermediate levels (combined for the analysis), and $31 \%$ on the bottom level of every branch in the structure. Some of the H-groups reconstructed the information structure as a network structure in the memory tests.

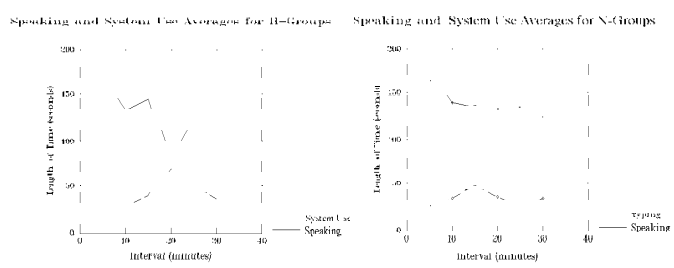

Figures 3a and 3b: Distribution averages of speaking and system use for $\mathrm{H}$ and $\mathrm{N}$ conditions

Group participation. Since speaking and typing were coded in seconds, in order to be as precise as possible, we used only the first 30 minutes from all groups after brainstorming was finished, and eliminated two groups due to minor system errors. Since we did not feel that the other results were affected by the errors, we elected to keep the results for the other analyses. No difference was found between conditions in the absolute amount of participation time for speaking or system use. However, we did find interesting results when speaking and system use were plotted in five minute intervals. Figures $3 a$ and $3 b$ show the averages for both conditions, for speaking and system use. For the H-groups, speaking decreases and system use increases in one of the five minute intervals, generally occurring around 20 minutes into the structuring task (Figure 3a). In contrast, the amount of speaking and system use in the $\mathrm{N}$-groups generally follows a uniform distribution (Figure 3b). One sees the correspondence between the dip and peak in the group averages for the H-groups but no clear correspondence appears in the curves for the $\mathrm{N}$-group averages.

Group satisfaction. N-groups reported higher satisfaction working in their groups than H-groups $(\mathrm{t}(14)=2.22, \mathrm{p}<.03)$. 


\section{Interpretation of results and future work}

\subsection{Interpretation of results}

Based on the group protocols, combined with the quanititative measures, a common thread appears to emerge to bind together some of the results of the $\mathrm{H}$ groups. We argue that the hypermedia functionality of creating nodes, adding content, and linking nodes affects the group process, strategy, and the nature of the documents produced. This capability is in contrast to working on a 2-D "flat" working surface. We now address the four questions raised earlier in Section 2. Hypermedia document creation: for groups as well as for individuals?

We found that with a reasonable training period all the groups were able to use the hypermedia structures without difficulty. Despite the fact that a nonlinear structure was not a requirement of the information structuring, six out of the eight $\mathrm{H}$-groups created a nonlinear structure using inter-node links, showing that groups understood the concept of nonlinearity. One $\mathrm{N}$-group also created a nonlinear structure using handdrawn arrows (see Figure 1).

Does hypermedia facilitate the formation and elaboration of relationships?

$\mathrm{H}$-groups produced documents with more relationships and deeper elaborations of concepts, which confirmed our expectation. Hypermedia use resulted in documents with more levels of depth, and with a greater number of ideas associated with the superordinate concepts. The functionality of creating nodes could explain the deeper levels because of the availability of sorting information into separate nodes and the ease of its access. Adopting a metaphor of a book, one group proposed putting the superordinate ideas on the first "page" and then putting the subordinate ideas on a second "page". The greater number of ideas associated with the superordinate concepts follows as a result of the deeper levels since they generally led to wider branches at a lower level in the tree structure.

Groups who used hypermedia were more likely to create network, rather than pure hierarchical structures, and included more ideas in the structures as well. In addition, their ideas and problem approach were judged by experts to be more original. In a similar argument to the availability of the node structure, the prevalence of the network structures could have been due to the availability of the link function, as opposed to the N-condition, where groups must hand-draw arrows in order to indicate a relationship. Links were also used to create unexpected relationships. One group used links between the superordinates to establish a "higher" and "lower" level of superordinates, although they were all contained on the same page.

The claim that the complexity of relationships produced using hypermedia may facilitate problem solving can also be explained by considering some of the models developed in cognitive psychology. Anderson (1983) claims that memory is organized as a network structure and that the spread of activation among associated concepts is an automatic process. This view is consistent with the facilitation of a hypertext-based network representation and problem solving.

How does hypermedia influence strategy and memory? 
$\mathrm{H}$-groups were more likely to use a top-down strategy whereas $\mathrm{N}$-groups tended to develop one superordinate concept at a time. One explanation could be that $\mathrm{H}$ groups knew that they had unlimited space in depth, which could be considered as a "third dimension" by creating and nesting nodes many levels down. Thus, they could plan the top level knowing they would have room for as many subordinate concepts as they needed. Some of the H-groups referred to creating and opening nodes as a space-keeping device which supports this notion. In one group, one member suggested that they put the brainstorming results in a new node, as a "notebook", to create a free page to plan the superordinate concepts. After the group had decided on the superordinate concepts, one member proposed that they create "subordinate" nodes.

A memory test revealed the $\mathrm{N}$-groups to recall more of the total information than the $\mathrm{H}$-groups, against our expectations. The $\mathrm{N}$-groups could have recalled more information because they viewed all of the information a longer amount of time compared to the H-groups who kept changing the information on their screen by opening and closing different nodes. However, although $\mathrm{H}$-groups viewed the superordinate concepts for less time, they recalled them as well as the $\mathrm{N}$-groups.

How does hypermedia influence group participation and satisfaction?

The speaking and system use over time of the H-groups show a trend for a different temporal work pattern than for the N-groups. Temporal patterning in groups is described as involving sets of complex activities including work flow coordination, matching time allocation to tasks, and synchronization of group members (McGrath, 1993) as well as involving conversation patterns (Mark, 1992). Whereas N-groups distribute their speaking and system use generally equally throughout the meeting, $\mathrm{H}$-groups tend to work in a more uneven pattern.

The five-minute interval where system use increases in the H-groups appears to be related to the use of the top-down strategy. Many of the H-groups had one member who proposed a plan of action which appears to have been a catalyst for the group to take action. Speaking declined and system use increased as a result of the group carrying out this plan. There was some variability however, concerning the proposals, such as whether a proposal was acted upon immediately or discussed, or the number of proposals concerning the action. In five of the groups, the proposal was for the group to divide into subgroups and work in parallel, and in one group the proposal was for collective work. For example, in group $\mathrm{H} 1$, one member proposed that now that they have the superordinates, they begin to divide up the subpoints. During the peak in system use, one member worked on the Liveboard to turn the superordinates into nodes and created the network structure by linking the superordinates. After a five-minute period, speaking increased due to discussing details of how the subordinates should be categorized. In group H3, the members decided to contrast an electronic with a conventional library. One member proposed that they break up into subgroups. One subgroup could work on the conventional, and the other on the electronic. The group then divided up, and this work pattern is reflected in the system use peak. Group H5, after reviewing their brainstormed ideas, decided to divide the ideas up into three work areas: building, offerings, and organization. One member proposed that they begin sorting the ideas into these three areas (corresponding to 
the three group members), and the group divided up to begin working, again reflecting this system use peak. Group $\mathrm{H} 7$ proposed to divide up into two subgroups--one responsible for technology, and the other responsible for the building. However, the group began to work on technology and the building, but did so publicly using the Liveboard, ignoring the proposal for parallel work. Again, this action is reflected in the system use peak. In group $\mathrm{H} 4$, a member proposed that the group now work independently. Each group member chose a superordinate to develop and the system use peak reflects the time when members first began to work separately.

Thus, a summary of a typical group process using the hypermedia functionality can be described as follows. Groups used a top-down strategy, and spent the first part of the organizing session planning what superordinate concepts to create. Then, a proposal was made for the group to divide up the superordinate concepts and work on them individually, which the members then did (four of the groups worked in parallel at this point; two of the groups performed the sorting operation publicly). The peak in system use and dip in speaking seems to reflect this action. After an initial period of elaborating the superordinates, the group began to discuss new points, generally clarification, e.g. verifying what the other subgroup had done, or under which superordinate a particular idea would be more appropriate.

$\mathrm{H}$-groups reported a lower satisfaction working in the group than $\mathrm{N}$-groups. In the questionnaire, H-groups reported that DOLPHIN was significantly harder to use than the $\mathrm{N}$-groups $(\mathrm{p}<.05)$, a result not surprising considering the added functionality of the hypermedia, and which could partially explain their lower satisfaction. The lower group satisfaction in the H-condition is consistent with other results found when groups use a new technology (McLeod, 1992; Olson et al., 1993). Using the electronic whiteboard may be analogous to using familiar markers on a whiteboard, and this may have resulted in higher satisfaction for $\mathrm{N}$ groups. We assume that overcoming an initial period of getting familiar with a new technology would lead to higher satisfaction. Despite their lower satisfaction, however, H-groups produced a more original and elaborate product.

\subsection{Comparisons to other studies and future work}

Our results suggest that when groups use hypermedia in problem solving, they change their habits, i.e., creating more deeply elaborated and more networkoriented problem representations. Our N-condition is similar to Tivoli (Pedersen et al., 1993). Therefore, it seems plausible to expect similar differences in group process and product if DOLPHIN use were to be compared to Tivoli use.

Some studies have addressed the impact of technology on groups in face-toface meetings (e.g. for a meta-analysis see McLeod, 1992; or for the impact of a simple collaborative editor ShrEdit, see Olson et al. 1993). We feel that our study extends this direction by looking at finer distinctions between groups who use different forms of technology. Although in the NICK experiment (Rein \& Ellis, 1989), the use of an electronic blackboard was contrasted with the use of 
networked workstations (resulting in more group exchange and group focus with higher group product ratings for the electronic blackboard condition), we looked at the combined effect of Liveboard with networked workstations. The studies we know of fall short in terms of comparing the impact of different kinds of software as we did by examining the added value of providing hypermedia functionality.

Complementing this work on early phases, we plan to investigate later phases of problem solving, as well as medium range and longterm collaboration, public vs. private work, and hypermedia as a "medium" for coordination. We plan to do this in more realistic situations, e.g., in the business domain. Our results suggest research opportunities for the future development of DOLPHIN. For example, in the redesign of DOLPHIN we want to include new support for orientation and navigation in the hypermedia structures. In another direction, we will investigate the use of DOLPHIN to include "virtual meetings" by coupling remote meeting rooms and external experts via ATM-based networks (Streitz et al., 1994).

\section{Acknowledgments}

The authors would like to thank the following people for their invaluable help and support: Jörg Geißler, Petra Rexroth, Chris Neuwirth, Matthias Will, Elke Teich, Adelheit Stein, Wiebke Möhr, Ajit Bapat, and finally Lutz Kirchner, Christian Schuckmann, and Jan Schümmer.

\section{References}

Anderson, J. (1983). The Architecture of Cognition. Cambridge: Harvard University Press.

Craik, F., Lockhart, R. (1972). Levels of processing: a framework for memory research. Journal of Verbal Learning and Verbal Behavior, 11, 671-684.

Elrod, S. et al. (1992). Liveboard: a large interactive display supporting group meetings, presentations and remote collaboration. Proc. of the CHI'92 Conf., Monterey,, pp. 599-607.

Gick, M., Holyoak, K. (1983). Schema induction and analogical transfer. Cognitive Psychology., $15,1-38$.

Grønbaek, K., Hem, J., Madsen, O., Sloth, L. (1994) Cooperative hypermedia systems: A Dexterbased architecture. Communications of the ACM, 37 (2): 64-74.

Haake, J., Neuwirth, C., Streitz, N. (1994). Coexistence and transformation of informal and formal structures: Requirements for more flexible hypermedia systems. Proc. of ACM European Conference on Hypermedia Technology (ECHT'94). Edinburgh, pp. 1-12.

Ishii, H., Kobayashi, M. Grudin, J. (1993). Integration of interpersonal space and shared workspace. ACM Transactions on Information Systems, Special issue on CSCW. T. Malone \& N. Streitz (Eds.), 11(4), 349-375.

Landow, G. (1989). Hypertext in literary education, criticism, and scholarship. Computers and the Humanities, 23, 173 - 198.

Mantei, M. (1988). Capturing the Capture Lab Concepts: A case study in th design of computersupported meeting rooms. Proc. of the ACM Conf. on CSCW '88, Portland, 257-268.

Mark, G. (1992). The generation of idea proposals: an illustration in a negotiation setting. Behavioral Decision-Making Research in Management Conference, UC, Berkeley. EDS Center for Advanced Research Technical Report.

Marmolin, H., Sundblad, Y., Pehrson, B. (1991). An analysis of design and collaboration in a distributed environment Proc. 2. European Conference on CSCW, Amsterdam. pp. 147-162. 
Mayer, R, Greeno, J. (1975). Effects of meaningfulness and organization on problem solving and computability judgements. Memory \& Cognition, 3, $356-362$.

McGrath, J. E. (1993). Time, interaction, and performance (TIP). In R. Baecker (Ed.), Groupware and Computer-Supported Cooperative Work, San Mateo: Morgan Kaufman, pp. 116-129.

McGuffin, L. \& Olson, G. M. (1992). ShrEdit: A Shared Electronic Workspace. Technical Report No. 45, University of Michigan, Cognitive Sciences and Machine Intelligence Lab, 1992.

McLeod, P. (1992). An assessment of the experimental literature on electronic support of group work. Human Computer Interaction, 7, 257 - 280.

Newell, A. (1980). Reasoning, problem solving, and decision processes: The problem space as the fundamental category. In R. Nickerson (Ed.), Attention and Performance VIII.. Erlbaum.

Nielsen, J. (1995). Multimedia and Hypertext: The Internet and Beyond. NY: Academic Press.

Nunamaker, J.F. et al. (1991). Electronic meeting systems to support group work. Communications of the ACM, 34(7):40-61.

Olson, J., Olson, G., Storrosten,M., Carter, M. (1993). Groupwork close up: A comparison of the group design process with and without a simple group editor. ACM Transactions on Information Systems, Special issue on CSCW, T. Malone \& N. Streitz (Eds.). 11(4):321-348.

Pedersen, E., McCall, K., Moran, T., Halasz, F. (1993). Tivoli: An electronic whiteboard for informal workgroup meetings, Proc. of the InterCHI'93 Conf., Amsterdam, pp. 391-398.

Rein , G. L., Ellis, C. A. (1989). The Nick experiment reinterpreted: Implications for developers and evaluators of groupware. Office: Technology and People, 5(1):47-75.

Osborn, A. F. (1957). Applied Imagination. New York: Charles Schribner's Sons.

Schuler, W., Hannemann, J., Streitz, N. (Eds.) (1995). Designing User Interfaces for Hypermedia. Heidelberg: Springer.

Schwartz, S. (1971). Modes of representation and problem solving: Well evolved is half solved. Journal of Experimental Psychology, 91, 347 - 350.

Stefik, M. et al. (1987). Beyond the chalkboard: computer support for collaboration and problem solving in meetings. Communications of the ACM, 30(1), 32-47.

Streitz, N. (1994). Putting objects to work: Hypermedia as the subject matter and the medium for computer-supported cooperative work. In M. Tokoro \& R. Pareschi (Eds.), Object-Oriented Programming. (ECOOP'94). Lecture Notes in Computer Science. Springer, pp. 183-193.

Streitz, N., Geißler, J. Haake, J., Hol, J. (1994). DOLPHIN: Integrated meeting support across Liveboards, local and remote desktop environments. Proc. of the ACM Conf. on ComputerSupported Cooperative Work (CSCW'94) Chapel Hill, pp. 345 - 358.

Streitz, N., Haake, J., Hannemann, J., Lemke, A., Schuler, W., Schütt, H., Thüring, M. (1992). SEPIA: A cooperative hypermedia authoring environment. Proc. of the 4th ACM European Conf. on Hypertext (ECHT'92), Milan, pp. 11-22.

Streitz, N. (1985). Subjektive Wissensrepräsentationen als Determinanten kognitiver Prozesse. Doctoral Dissertation. Technical University of Aachen.

Wingfield, A., Byrnes, D. (1981). The psychology of human memory. NY: Academic Press. 